\title{
PRIVATE AUSGABEN FÜR DIE TERTIÄRE BILDUNG
}

Die Bildungseinrichtungen der OECD-Länder werden zwar überwiegend mit öffentlichen Mitteln finanziert, der Anteil der privaten Finanzierung im Tertiärbereich ist jedoch erheblich und weiter im Steigen begriffen. In diesem Bildungsbereich wird die Beteiligung des Einzelnen und anderer privater Akteure an den Bildungskosten zunehmend als ein wirksamer Weg betrachtet, um sicherzustellen, dass für Studierende, unabhängig von ihrem sozioökonomischen Hintergrund, Finanzierungsmittel zur Verfügung stehen.

\section{Definition}

Dieser Indikator umfasst die privaten Ausgaben für Schulen, Hochschulen und andere private Einrichtungen, die Bildungs-

\section{Überblick}

In allen Ländern ist der Anteil der privaten Bildungsausgaben im Tertiärbereich - mit durchschnittlich 30\% der Gesamtausgaben - wesentlich höher als im Primar-, Sekundar- und postsekundären nichttertiären Bereich (9\%). Der von Privatpersonen, Unternehmen und anderen privaten Akteuren getragene Anteil der Ausgaben für Einrichtungen des Tertiärbereichs, einschließlich bezuschusster privater Zahlungen, liegt zwischen weniger als $5 \%$ in Dänemark, Finnland und Norwegen und mehr als $40 \%$ in Australien, Israel, Japan und den Vereinigten Staaten sowie über $70 \%$ in Chile, Korea und dem Vereinigten Königreich. Unter diesen Ländern sind in Korea und im Vereinigten Königreich die meisten Studierenden in privaten Einrichtungen eingeschrieben (rd. $80 \%$ an privaten Hochschulen in Korea; $100 \%$ an vom Staat abhängigen privaten Einrichtungen im Vereinigten Königreich), und ein Großteil der Mittel der Bildungseinrichtungen stammt aus Studiengebühren (über $49 \%$ in Korea und über 58\% im Vereinigten Königreich).

Im Durchschnitt der OECD-Länder ist der Beitrag privater Akteure (ohne private Haushalte) zur Finanzierung der Bildungseinrichtungen im tertiären Bildungsbereich höher als in anderen Bildungsbereichen. In Australien, Kanada, der Tschechischen Republik, Israel, Japan, Korea, den Niederlanden, der Slowakischen Republik, Schweden, im Vereinigten Königreich und in den Vereinigten Staaten werden $10 \%$ oder mehr der Ausgaben für Einrichtungen des Tertiärbereichs von privaten Akteuren (ohne private Haushalte) getragen. In Schweden dienen diese Beiträge beispielsweise großenteils der Förderung von Forschung und Entwicklung.

Zwischen 2000 und 2009 verzeichneten 18 der 25 Länder, für die vergleichbare Daten verfügbar sind, einen Anstieg des Anteils der privaten Finanzierungen für den tertiären Bildungsbereich. Dieser Anteil erhöhte sich durchschnittlich um 7 Prozentpunkte, und um über 10 Prozentpunkte in Mexiko, Portugal, der Slowakischen Republik und dem Vereinigten Königreich (wo sich der Anteil um 38 Prozentpunkte erhöhte). Während der Anteil der privaten Finanzierungen für den tertiären Bildungsbereich in einigen Ländern in diesem Zeitraum erheblich stieg, war dies in anderen Bildungsbereichen nicht der Fall. dienstleistungen erbringen oder unterstützen. $\mathrm{Zu}$ den anderen privaten Akteuren zählen Privatunternehmen und Einrichtungen ohne Erwerbscharakter, wie z.B. kirchliche Organisationen und Wohltätigkeitsvereine sowie Arbeitgeber- und Arbeitnehmervereinigungen. Die Ausgaben privater Unternehmen für die betriebliche Komponente der dualen schulischen und betrieblichen Ausbildung von Berufsauszubildenden und Studierenden sind darin ebenfalls berücksichtigt.

Die privaten Ausgaben werden abzüglich öffentlicher Subventionen für Zahlungen an Bildungseinrichtungen erfasst. Inbegriffen sind auch außerhalb von Bildungseinrichtungen getätigte Bildungsausgaben.

\section{Vergleichbarkeit}

Die Ausgabendaten wurden im Rahmen einer im Jahr 2011 durchgeführten Erhebung ermittelt, bei der konsistente Methoden und Definitionen zum Einsatz kamen. Die Ausgabendaten basieren auf den Definitionen und dem Erfassungsbereich der UNESCO/OECD/Eurostat-Datenerhebung zur Bildung; sie wurden mit Hilfe des BIP-Preisdeflators an das Preisniveau von 2009 angepasst. Die Verwendung von Daten und Definitionen aus einer einheitlichen Erhebung gewährleistet eine gute Vergleichbarkeit der Ergebnisse zwischen den einzelnen Ländern.

\section{Quelle}

- OECD (2012), Bildung auf einen Blick, W. Bertelsmann Verlag, Bielefeld.

\section{Weitere Informationen}

\section{Analysen}

- OECD (2013), Trends Shaping Education, OECD Publishing.

- OECD (2012), Highlights from Education at a Glance, OECD Publishing.

- OECD (2012), Higher Education Management and Policy, OECD Publishing.

- OECD (2012), Reviews of National Policies for Education, OECD Publishing.

\section{Zur Methodik}

- OECD (2004), OECD Handbook for Internationally Comparative Education Statistics: Concepts, Standards, Definitions and Classifications, OECD Publishing.

- UNESCO Institute for Statistics (UIS), OECD und Eurostat (2011), UOE Data Collection on Education Systems, UIS, Montreal.

\section{Online-Datenbanken}

- OECD Education Statistics.

Websites

- OECD Education at a Glance, www.oecd.org/edu/eag2012. 
Öffentliche und private Ausgaben für Bildungseinrichtungen im Tertiärbereich

\begin{tabular}{|c|c|c|c|c|c|c|c|c|c|}
\hline & \multicolumn{7}{|c|}{ In Prozent der Gesamtausgaben } & \multicolumn{2}{|c|}{ Index $2000=100$} \\
\hline & \multicolumn{2}{|c|}{ Öffentliche Mittel } & \multicolumn{5}{|c|}{ Private Mittel } & \multirow{3}{*}{$\begin{array}{l}\text { Öffentliche Mittel } \\
2009 \text { oder letztes } \\
\text { verfügbares Jahr }\end{array}$} & \multirow{3}{*}{$\begin{array}{c}\text { Private Mittel } \\
\\
2009 \text { oder letztes } \\
\text { verfügbares Jahr }\end{array}$} \\
\hline & \multirow{2}{*}{2000} & \multirow{2}{*}{$\begin{array}{l}2009 \text { oder letztes } \\
\text { verfügbares Jahr }\end{array}$} & Insgesamt & $\begin{array}{l}\text { Ausgaben privater } \\
\text { Haushalte }\end{array}$ & Sonstige & Insgesamt & Davon: subventioniert & & \\
\hline & & & 2000 & $\begin{array}{l}2009 \text { oder letztes } \\
\text { verfügbares Jahr }\end{array}$ & $\begin{array}{l}2009 \text { oder letztes } \\
\text { verfügbares Jahr }\end{array}$ & $\begin{array}{l}2009 \text { oder letztes } \\
\text { verfügbares Jahr }\end{array}$ & $\begin{array}{l}2009 \text { oder letztes } \\
\text { verfügbares Jahr }\end{array}$ & & \\
\hline Australien & 49.9 & 45.4 & 50.1 & 39.1 & 15.4 & 54.6 & 0.5 & 135.0 & 161.4 \\
\hline Belgien & 91.5 & 89.7 & 8.5 & 5.5 & 4.8 & 10.3 & 3.9 & 122.6 & 150.5 \\
\hline Chile & .. & 23.4 & .. & 68.1 & 8.5 & 76.6 & 9.3 & .. & .. \\
\hline Dänemark & 97.6 & 95.4 & 2.4 & .. & .. & 4.6 & .. & 120.7 & 236.4 \\
\hline Deutschland & 88.2 & 84.4 & 11.8 & & .. & 15.6 & .. & 119.9 & 166.1 \\
\hline Estland & .. & 80.2 & .. & 18.2 & 1.6 & 19.8 & .. & 163.3 & .. \\
\hline Finnland & 97.2 & 95.8 & 2.8 & .. & .. & 4.2 & .. & 127.3 & 198.0 \\
\hline Frankreich & 84.4 & 83.1 & 15.6 & 9.7 & 7.3 & 16.9 & .. & 121.7 & 134.3 \\
\hline Griechenland & 99.7 & .. & 0.3 & .. & .. & .. & .. &.. & .. \\
\hline Irland & 79.2 & 83.8 & 20.8 & 13.8 & 2.4 & 16.2 & .. & 151.5 & 111.3 \\
\hline Island & 91.8 & 92.0 & 8.2 & 7.4 & 0.6 & 8.0 & .. & 159.4 & 154.5 \\
\hline Israel & 58.5 & 58.2 & 41.5 & 27.3 & 14.6 & 41.8 & 5.0 & 107.8 & 109.3 \\
\hline Italien & 77.5 & 68.6 & 22.5 & 23.8 & 7.6 & 31.4 & 8.5 & 103.9 & 164.4 \\
\hline Japan & 38.5 & 35.3 & 61.5 & 50.7 & 14.1 & 64.7 & .. & 104.8 & 120.3 \\
\hline Kanada & 61.0 & 62.9 & 39.0 & 20.2 & 16.9 & 37.1 & .. & 130.5 & 120.5 \\
\hline Korea & 23.3 & 26.1 & 76.7 & 49.2 & 24.8 & 73.9 & 1.4 & 182.9 & 157.5 \\
\hline Luxemburg & .. & & .. & & .. & .. & & .. & .. \\
\hline Mexiko & 79.4 & 68.7 & 20.6 & 30.9 & 0.4 & 31.3 & 1.8 & 145.9 & 256.3 \\
\hline Neuseeland & .. & 67.9 & .. & 32.1 & .. & 32.1 & .. & 157.2 & .. \\
\hline Niederlande & 76.5 & 72.0 & 23.5 & 14.9 & 13.1 & 28.0 & 0.4 & 126.9 & 160.9 \\
\hline Norwegen & 96.3 & 96.1 & 3.7 & 3.0 & .. & 3.9 & .. & 123.6 & 130.6 \\
\hline Österreich & 96.3 & 87.7 & 3.7 & 2.9 & 9.4 & 12.3 & 8.8 & 142.2 & 517.7 \\
\hline Polen & 66.6 & 69.7 & 33.4 & 22.8 & 7.5 & 30.3 & .. & 211.4 & 182.7 \\
\hline Portugal & 92.5 & 70.9 & 7.5 & 22.3 & 6.8 & 29.1 & .. & 109.0 & 548.4 \\
\hline Schweden & 91.3 & 89.8 & 8.7 & .. & 10.2 & 10.2 & .. & 125.3 & 149.7 \\
\hline Schweiz & .. & & .. & & .. & .. & .. & 128.9 & .. \\
\hline Slowak. Rep. & 91.2 & 70.0 & 8.8 & 11.7 & 18.3 & 30.0 & 2.0 & 138.9 & 619.6 \\
\hline Slowenien & .. & 85.1 & .. & 10.8 & 4.2 & 14.9 & .. & .. & .. \\
\hline Spanien & 74.4 & 79.1 & 25.6 & 16.8 & 4.1 & 20.9 & 1.7 & 149.3 & 114.8 \\
\hline Tschech. Rep. & 85.4 & 79.9 & 14.6 & 8.8 & 11.3 & 20.1 & .. & 202.4 & 298.3 \\
\hline Türkei & 95.4 & .. & 4.6 & .. & .. & .. & .. &.. & .. \\
\hline Ungarn & 76.7 & .. & 23.3 & .. & .. & .. & .. & 134.7 & .. \\
\hline Ver. Königreich & 67.7 & 29.6 & 32.3 & 58.1 & 12.3 & 70.4 & 10.8 & 116.7 & 334.2 \\
\hline Ver. Staaten & 31.1 & 38.1 & 68.9 & 45.3 & 16.6 & 61.9 & .. & 137.5 & 100.9 \\
\hline EU27 & .. & .. & .. & .. & .. & .. & .. & .. & .. \\
\hline OECD & 77.1 & 70.0 & 22.9 & .. & .. & 30.0 & 3.2 & 138.0 & 215.9 \\
\hline Brasilien &.. &.. &.. &.. &.. &.. & .. & 162.5 &.. \\
\hline China & .. & .. & .. & .. & .. & .. & .. & .. & .. \\
\hline Indien & .. & & .. & & .. &.. & .. & .. & .. \\
\hline Indonesien & .. & & .. & & .. & .. & .. &.. & .. \\
\hline Russ. Föderation & .. & 64.6 &.. & 27.4 & 8.0 & 35.4 & .. & 378.8 & .. \\
\hline Südafrika & .. & .. & .. & .. & .. & .. & .. & .. & .. \\
\hline
\end{tabular}

StatLink Ails http://dx.doi.org/10.1787/888932709681

\section{Anteil der privaten Ausgaben für Bildungseinrichtungen im Tertiärbereich}

In Prozent

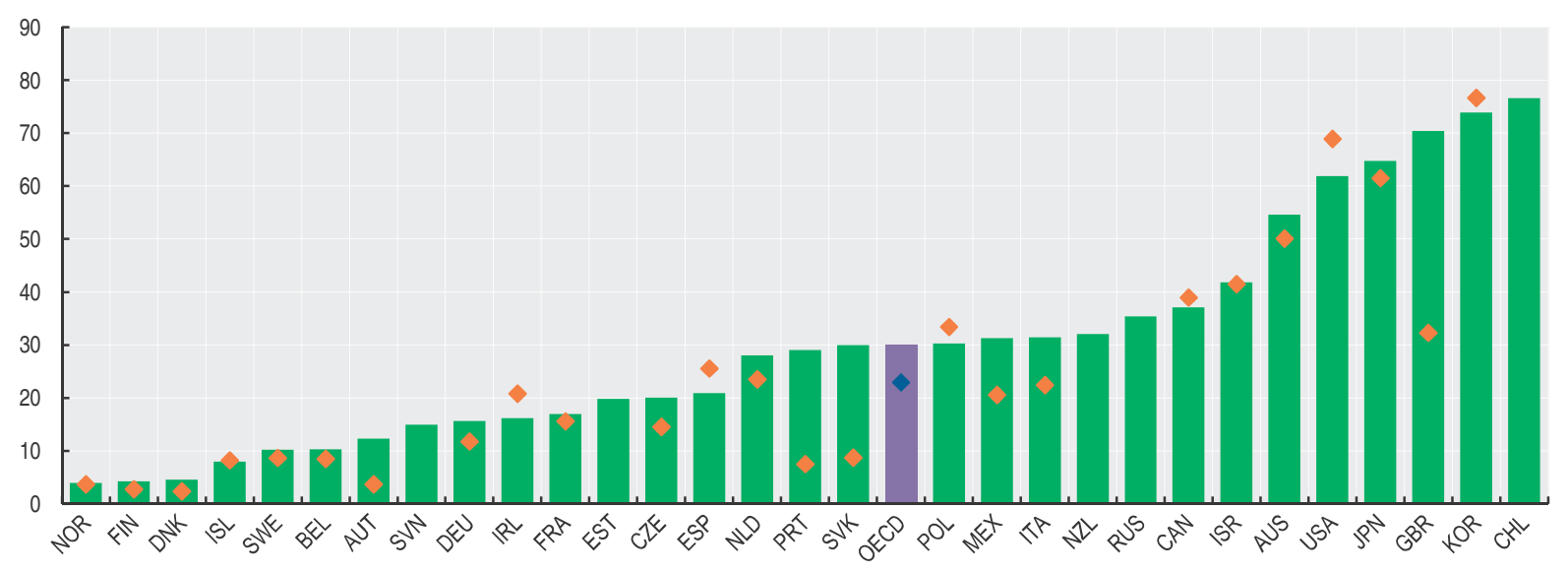




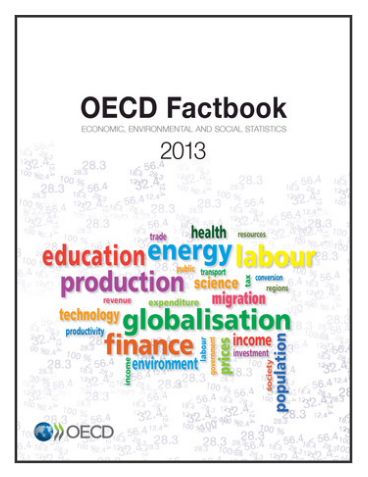

\author{
From: \\ OECD Factbook 2013 \\ Economic, Environmental and Social Statistics
}

Access the complete publication at:

https://doi.org/10.1787/factbook-2013-en

\title{
Please cite this chapter as:
}

OECD (2013), "Private Ausgaben für die tertiäre Bildung", in OECD Factbook 2013: Economic, Environmental and Social Statistics, OECD Publishing, Paris.

DOI: https://doi.org/10.1787/factbook-2013-79-de

Das vorliegende Dokument wird unter der Verantwortung des Generalsekretärs der OECD veröffentlicht. Die darin zum Ausdruck gebrachten Meinungen und Argumente spiegeln nicht zwangsläufig die offizielle Einstellung der OECDMitgliedstaaten wider.

This document and any map included herein are without prejudice to the status of or sovereignty over any territory, to the delimitation of international frontiers and boundaries and to the name of any territory, city or area.

You can copy, download or print OECD content for your own use, and you can include excerpts from OECD publications, databases and multimedia products in your own documents, presentations, blogs, websites and teaching materials, provided that suitable acknowledgment of OECD as source and copyright owner is given. All requests for public or commercial use and translation rights should be submitted to rights@oecd.org. Requests for permission to photocopy portions of this material for public or commercial use shall be addressed directly to the Copyright Clearance Center (CCC) at info@copyright.com or the Centre français d'exploitation du droit de copie (CFC) at contact@cfcopies.com. 